Témoigner Témoigner. Entre histoire et mémoire

Getuigen Revue pluridisciplinaire de la Fondation Auschwitz

$118 \mid 2014$

Au nom des victimes. Dictature et terreur d'État en Argentine, Chili et Uruguay

\title{
Victimes du souvenir et de l'oubli
}

Oblivion et la possibilité de déconstruire les processus de victimisation

Victims of remembrance and forgetfulness: Oblivion and the potentiality of

deconstructing processes of victimization

Herinneren en vergeten. Oblivion en de deconstructie van de slachtofferfiguur

\section{Susana Draper}

Traducteur : Antonia García Castro

\section{(2) OpenEdition}

12 Journals

\section{Édition électronique}

URL : http://journals.openedition.org/temoigner/961

DOI : 10.4000/temoigner.961

ISSN : 2506-6390

Éditeur :

Éditions du Centre d'études et de documentation Mémoire d'Auschwitz, Éditions Kimé

\section{Édition imprimée}

Date de publication : 1 octobre 2014

Pagination : 121-127

ISBN : 978-2-84174-674-3

ISSN : 2031-4183

Référence électronique

Susana Draper, «Victimes du souvenir et de l'oubli », Témoigner. Entre histoire et mémoire [En ligne],

118 | 2014, mis en ligne le 01 octobre 2015, consulté le 23 octobre 2020. URL : http://

journals.openedition.org/temoigner/961; DOI : https://doi.org/10.4000/temoigner.961 


\section{URUGUAY}

\section{Victimes du souvenir et de l'oubli}

Oblivion et la possibilité de déconstruire les processus de victimisation

$\rightarrow$ Par Susana Draper, Princeton University

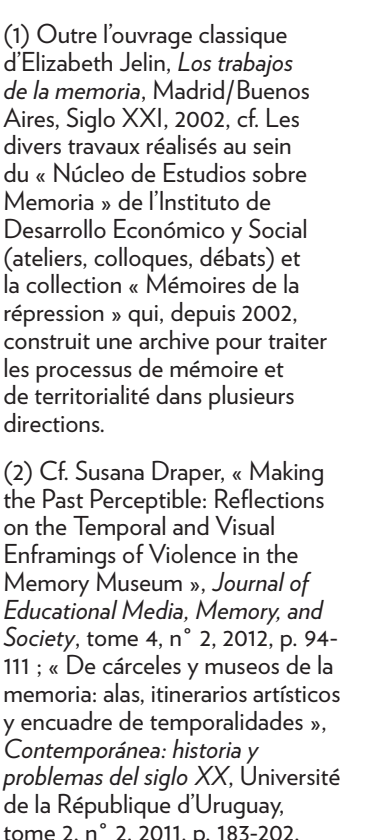

es interrogations qui ont guidé ce travail sont centrées sur la figure de la victime dans le contexte postdictatorial uruguayen en lien avec ce que nous appelons une topologie de la mémoire: l'articulation des mises en paroles de la mémoire avec diverses conceptions des territoires et des lieux (je pense surtout aux supports matériels, qu'il s’agisse d'endroits, de musées, de livres, etc.). Cette articulation de la parole et d'un lieu est considérée soit comme un moyen de s'exprimer au sujet d'espaces physiques déterminés, soit comme une référence à la construction d'une communauté centrée sur l'expérience de la remémoration' Je vais donc me centrer sur le cas uruguayen en mettant l'accent sur des expérences vécues en prison. Alors qu’en Argentine, la lutte pour la mémoire de la répression s'est surtout focalisée sur la pratique de la disparition forcée et de la possibilité de faire connaître le réseau des centres clandestins de détention et d'assassinat utilisès par les militaires, en Uruguay, c'est d'abord la question des prisonniers politiques et celle des divers sites d'emprisonnement qui ont pris de l'importance. Ce rôle centra de la prison dans les processus mémoriels liés à la répression sous la dictature

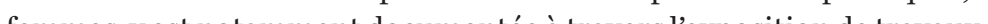
femmes, yestnotamn Ins ts qui ont été autant de formes de résistance à lexpérience carcérale ${ }^{2}$. Il s'agit de présenter comment la figure de la victime émerge dans l'espace textue féminin à partir d'une résistance étayée par des stéréotypes dominant le regard sur le passé. D’un côté, la figure hérö̈que du militant qui est tantôt celui qui se souvient, tantôt celui que l'on convoque dans un contexte épique connoté par le courage (associé au monde masculin). De l'autre, les victimes sans défense qui se souviennent ou dont on se souvient à partir d'une logique subjectiviste de la douleur et de l'impuissance (associée au monde féminin). La dichotomie entre la figure du militant et celle de lavictine siof de passé. Cette dichotomie porte sur le passé un regard qui postule des sujets pleins de 
URUGUAY

Victimes du souveni

et de lo
(suite)

(3) Edda Fabbri, Oblivion, La
Havane, Casa de las Américas 2007.

(4) Graciela Sapriza, « Memorias
de mujeres en el relato de la

dictadura (Uruguay, 1973-1985)

Deportate, esuli, profughe. Riviss
telematica di studi sulla memoria
femminile, Venise, $\mathrm{n}^{\circ} 11,2009$,

femminile,

(5) II existe bien d'autres

efump dancies químonoignent du

refus danciennes prisonnieres
politiques d'assumer le rôle de
«victimes » qui a continué à

«victimes » qui a continué à
leur être assigné dans le cadre

des politiques de mémoire de
'Éttat. Cf. Mariana Tello Weiss,

La ex cárcel del Buen Passor

en Córdoba: un territorio
de memorias en disputa, ,

España - Poratugare Berlin. Latina

bero-Amerikanischen Institut,
tome $X, n^{\circ} 40,2010$, p. 145-165,

Susana Draper, Afterlives of
Confinement Spatial Transitions

in Posstdictatorship Latin Americ

Pittsburghh, University of
Pittsburgh Press, 2012. force et de pouvoir (les héros) et d'autres qui n’ont eu aucune possibilité de résistance ni d'action. Or, la relation de pouvoir et la capacité de résistance ont été généralement plus complexes. L’approche que je propose est axée sur un texte spécifique Oblivion, écrit par une ancienne prisonnière politique, Edda Fabbri ${ }^{3}$. Cette œuvre aborde la thématique de la victimisation en opposant un double travail de résistance à cette logique binaire. Je voudrais analyser la déconstruction de cette bipolarité (héros-victime) par Fabbri en me penchant sur la façon dont elle structure la mise en scène du pascé : résister à la fois à la catécorie de militante hérö̈que et à celle de

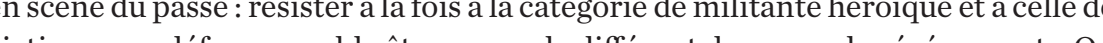
victime sans défense semble être un mode different de penser les evénements. On identifie, d'une part, une prise de distance vis-à-vis de ce que fut la lutte politique dans le passé et, d'autre part, la prédominance habituelle des hommes en leur qualité de militants révolutionnaires.

Ici, se situe le problème de la figure, comme construction sociale impliquan des sujets privés de capacité d'action. En d'autres termes, cela veut dire que l'on assiste à certaines formes de victimisation qui finissent par priver de nouveau les sujets victimisés de tout rôle actif, qu'il soit politique, historique ou social. En me centrant ici sur le texte d'Edda Fabbri, il me semble aussi important d'interroger la manière dont la différence de genre joue un rôle dans la caractérisation de la victime. Dans son étude sur la menoore des processu dictatoriaux à partir d'une perspective féninine, Graciela Sapriza souligne qu il existe une tendance à féminiser les processus de victimisation. On repère cela dans les «réferences commune du discours masculn » renvoyan aux « actions hérolques, risquées ou politiques dans le sens traditionnel ${ }^{4} »$. Ainsi, dès lors qu'il s'agit de victimisation, nous assistons généralement à un processus sémantique de féminisation des sujets, qu'ils soient hommes ou femmes. Néanmoins, il convient de noter que certaines ancienne prisonnières politiques interviennent dans le présent en remettant en question le processus de victimisation lui-même, et en rejetant également le discours de l'épopée et de l'héroïsme. Sapriza analyse des témoignages de femmes qui proposen des scénarios différents et mettent l'accent sur la vie quotidienne et les affects. Ces expressions alternatives sont puissantes: en problématisontla manière dontla figure de la victine peut reproduie un stérétype social (la fenme dépouvue doure de la victine peut reproduire un stéréotype social (la femme dépourvue d'outil pour lutter), la lutte politique du passé

\section{CONSTRUCTION SOCIALE : LA VICTIME COMME SUJET DE NON-POUVOIR?}

En quoi la figure de la victime est-elle problématique? Elle cristallise l'idée d'un sujet qui ne peut pas agir. Plus le pouvoir du bourreau est grand, plus la notion de victime prend de la force, mais cette force se fonde sur une impuissance, une incapacité à agir et à résister. Comment aborder dès lors les processus de victimisation et de leurs proies? politique des événements afin de prendre de la distance par rapport à ce modèle?

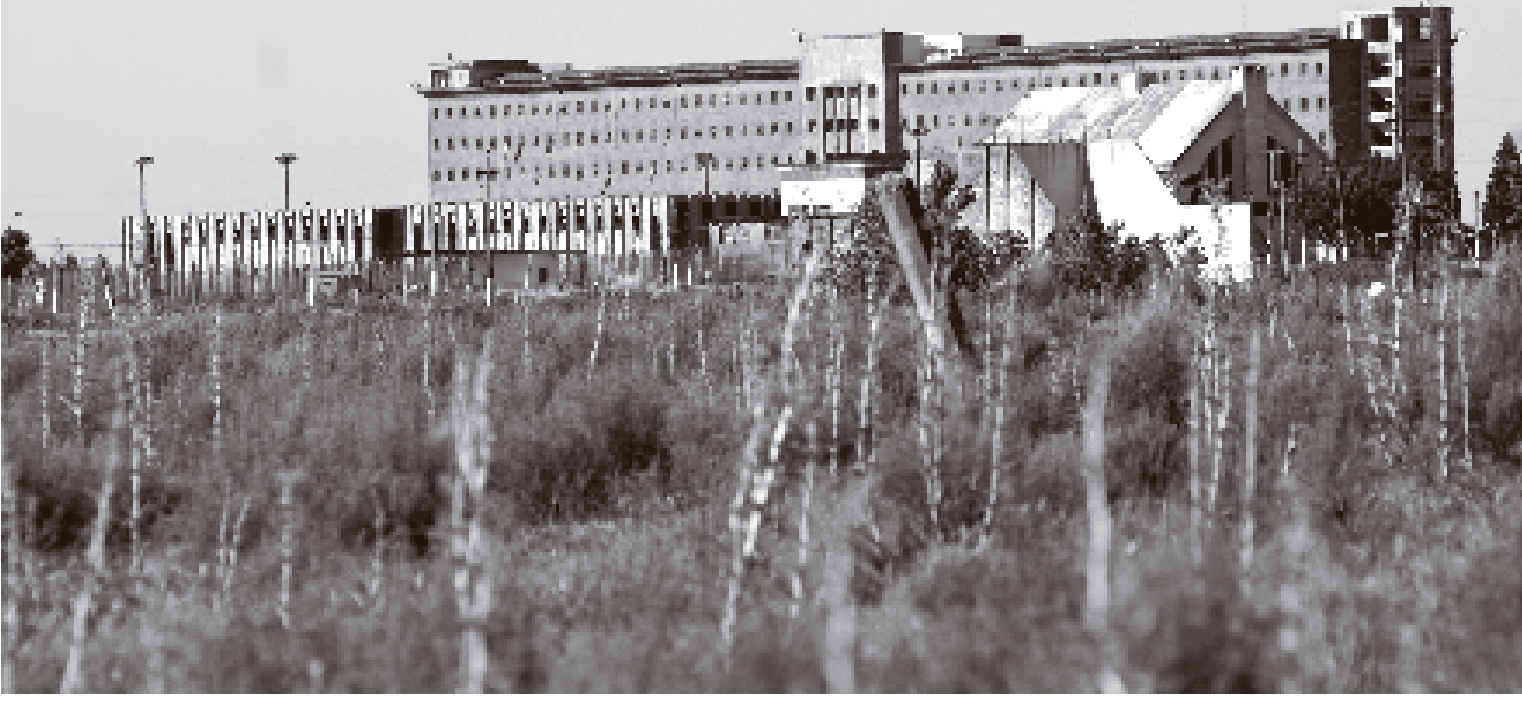

- Prison de Libertad

Voilà quelques-unes des questions qui guident le texte Oblivion et permettent de construire une approche à même de problématiser la mémoire, l'oubli et leur connexion avec le processus de victimisation.

Fabbri a été libérée en 1985. Presque vingt ans plus tard, elle écrit ce témoignage qui assume une double fonction : d'un côté, elle aborde son passé de prisonnière, de l'autre, elle commente de manière critique et créative le processus même de domestication de la mémoire qui a figé la figure de la victime. Sa réflexion sur le processus de victimisation surgit du travail d'écriture lui-même et engendre une sorte de critique performative. À partir de l'espace du témoignage, Fabbriquestionne une certaine automatisation du souvenir. C'est pourquoi Oblivion commence par une suspension de la figure du survivant devenue un cliché et nous fait entrer dans le silence trouble des questions : à quoi sert d'écrire sur le passé? Comment, de quel lieu du passé, le passé sécrit-il ? Quel type de dialogue entretient-il avec le présent? À quelle question du présent peut-il répondre?

Prisonnière politique de 1971 jusqu'au retour de la démocratie et de l'amnistie de 1985, détenue d'abord dans la prison du Cabildo, d'où elle s'est évadée avec 37 femmes, militantes tupamaras ${ }^{6}$, puis de celle de Punta de Rieles, Fabbri nous invite à penser le rôle de l'écriture dans l'élaboration du souvenir, et notamment à ne pas considérer comme automatiques des mécanismes qui se répètent à chaque fois que l'on tente de produire un témoignage sur le passé7. Il convient dès lors de se demander : comment agit le texte? Comment se constitue-t-il en acte de résistance et quelles en sont les implications?

Le titre même de l'œuvre, Oblivion pose le défi d'éluder des dichotomies implicites dans la figure de la victime, puisqu'il s’agit d'une recherche qui conçoit la mémoire dans la une modalité de réflexion sur un sujet désormais distant - la prisonnière con me passé et en luttant avec, contre toutes les médiations qui opèrent entre passé et à Montevideo (Uruguay).
(6) Le Movimiento de Liberación
Nacional-Tupamaros est mouvement politicue de de gave uruguayen qui prône la guérilla urbaine pendant les années 1960
et au début des années 1970 (7) Cf. Graciela Jorge, Eleuterio 13 palomas y 38 estrellas: fugas de la carcel de mujeres, Montevideo 
présent. Dans un entretien ${ }^{8}$, Fabbri raconte que c'est seulement lorsque le passé a été suffisamment loin derrière elle qu’elle a pu commencer à imaginer une vie littéraire une reconstruction de cette expérience et de cette femme quelle ne reconnaissai presque pas : c'est peut-être dans cette non-reconnaissance que naît la parole capable de reconstruire une expérience d'emprisonnement d'où le moi était absent ${ }^{9}$.

Lorsqu'elle essaye d'aller chercher à la source le souvenir de l'emprisonnement, Fabbri fait souvent référence à la polyphonie d'une vie sans subjectivité, sans espace pour un moi en solitude. La vie en prison se présente comme pure collectivité mace pour quee par uncontrole permanent. Elle émerge textuellement comme vie sansécriture allexception de au texte - écrit bien des années plus tard - est celle de ses camarades de prison chantant, racontant des histoires, dansant, criant au milieu de la folie. C'est ains qu’au début de son livre, les voix du passé entrent dans le moment présent comme des échos de visiteurs.

Dans l'entretien déjà cité, Fabbri soutient que la mémoire est un droit, mais que l'oubli est une capacité. Dans la logique du texte, cela opère comme puissance transformatrice, lui permettant de commenter de manière critique les avatars de l'excès d'intérêt pour la mémoire des victimes et le manque de questions sur la manière

[...] je ne sais pas qui écrit l'histoire ni comment... Nous disons de vieilles phrases, tristes à force dềtre usées. Nous disions autrefois que l'histoire était le fait des vainqueurs, qưil fallait donc en écrire une autre, la vraie. Raconter la vérité pour que l'on sache, on dit ça maintenant, pour ne pas répéter le passé. Transmettre avant de mourir, laisser un héritage, disent les autres. Ces mots m’échappent; ils ne m’appartiennent en aucune façon, ils sonnent creux. Je n'ai aucun message à transmettre, en tous cas pas ce message (que je suis sensée savoir et qưun autre est sensé vouloir entendre). Mirza, Fernando, a dit que l'attitude c'est le seul message; l'écoute, quelque chose comme ça. Je ne veux plus m'interroger sur le message. Quand est-ce que nous allons nous interroger sur un silence? Ougnd

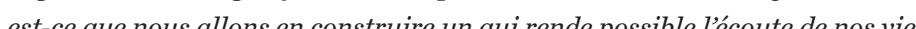
est ce que et desnoules $2 J$ in dite je le ré

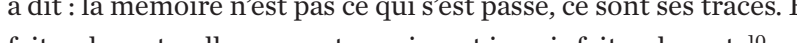
faites de mots, elles ne sont quasiment jamais faites de $\operatorname{mots}^{10}$.

Dans cet extrait, Fabbri présente comme cliché l'idée selon laquelle raconter le passé permet de ne pas répeter 'histoire - en jouant avec la manière dont le discours mémoriel (ce quej'ai appelé le tournant mémorialiste) est devenu un automatisme. En prenant position contre cette automatisation, Fabbri semble dire que toutes ces paroles automatisées finissent par faire taire ou faire mourir la mélodie du passé et ne laissent pas entendre le silence qui la compose (ses instants d'incertitude, sa douleur, son iŕvén côté lobsession pour rappeler et interroger le passé, à aborder les questions non (10) Edda Fabbri, Oblivion, op.
cit., p. 58 - c'est moi qui souligne. résolues, notamment celle de l'alternative à la violence qui existait probablement à l'époque. Fabbri évoque ainsi la possibilité de déconstruire le récit victimaire et la notion de sujet telle qu’elle est présentée dans les souvenirs des survivants et d'examiner ce qui, au cours de ce passé, est effectivement arrivéà ces jeunes femmes.

À la différence des textes masculins qui ont d’abord raconté les expériences militantes et l'emprisonnement, il y a ici une volonté de souligner la dimension collective du vécu de la prison ${ }^{11}$. Le sujet tend à s'effacer, le souvenir se focalisant sur ce qu’a été la vie en commun, comme si par l'écriture - dont Fabbri signale l'absence en prison-l'exercicemennedelarer collectif. Il est particulièrement intéressant d'analyser l'accent que de nombreux textes écrits par des femmes mettent sur la relation entre le langage (commun à des femmes de differentes cheses sociales (les violences sexuelles, la libération et les blocages moraux), le travail organisé en prison, la maternité et l'expérience ludique (chanter, représenter, dramatiser). Ces formulations des expériences du passé rendent compte d'un rêve de communauté et de la possibilité de partager la dimension incommensurable de ces souvenirs. Il faut probablement envisager ces données en lien avec ce qu’a été l'expérience de la vie militante pour ces femmes (la première génération de femmes à sêtre mobilisée politiquement de manière massive).

Dans l'écriture de la prison, le langage devient un espace de refuge, mais auss la haine, le sentiment de rejet envers un autre trop different - par exemple les prisonniers de droit commun -, le désir dévasion ainsi que la volonté de constituer un collectif qui partage le plus intime, cela même qui est anéanti par la prison : le geste, le $\operatorname{rir}^{12} \ldots$...

\section{LA MÉMOIRE AU PRÉSENT}

Depuis quelques années, aussi bien en Uruguay qu’en Argentine, de plus en plus de textes traitent de l'expérience des femmes pendant la dictature Leur spectre est de textes tón arge, allant du tem lexperie mémoire dans l'acte même de lecriture. Dans Tiempo pasado, Beatriz Sarlo ${ }^{13}$ oppose la mémoire subjectiviste à celle d'un champ plus intellectuel où la reconstruction du souvenir se fait à partir d'un essai de conceptualisation ${ }^{14}$. Sans adhérer complètement à cette division, qui d'une certaine manière court le risque de répéter une dichotomie entre sentiment et connaissance, entre subjectivité et objectivité, il est également important de penser la remémoration au prisme de ces dichotomies inhérentes au souvenir et son écriture.

Des textes comme celui de Fabbri confèrent à la narratrice un statut étrange. En effet, plusieurs années après l'expérience, elle tente d'élaborer une approche du passéquiprend en compte le problème de la distance temporelle. Le tempspasséest pansi envisé depuis le début comme une donnée centrale qui affecte le processé ainsi de remén
(11) Parmi les textes auxquels la plus grande diffusion Eleuterio Fernández Huidobro 3 vol., Montevideo, Tae 1986 et Mauricio Rosencof, Eleuterio Fernández Huidobro, Memon del calabozo, Buenos
Aguilar 2008 [1987].

(12) La dimension collective de ts résistance pen las dhumiliation les plus intenses apparất dans presque tous les
récits de lemprisonnement.

(13) Beatriz Sarlo, Tiempo pasado subjera de la memoria y giro
Xxtivo, Buenos Aires, Siglo

$X X I, 2005$ (14) On trouve des exemples
dans les textes de Pilar Calveiro, Pourcit et disparition. Les Camps traduit de lespagnol par lsabelle Taudière, La Fabrique, Paris,
2006, et d'Emilio de Ipola, 2006, et d'Emilio de Ipola,

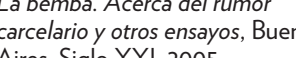


(lajeune femme) en ayant recours à la troisième personne, tantôt comme narratrice, à la première personne, tout en laissant émerger les dimensions problématiques de ce « je ». Le jeu que permet un récit sur la relation entre passé et présent conduit à une réflexion sur les deux figures que nous avons mentionnées plus haut : celle de héros d'une épopée et celle de victime. Le passage du temps est non seulement converti en dispositif narratif à partir duquel l'écrivain représente le passé, mais émerge aussi comme cadrage pour une interrogation menée à partir du présent.

Le texte est fortement critique envers les codes de conduite rigides, militarisés Leteste et stricts. Selon Fabbri, ces derniers empêchent de penser non seulementl'autre non assimilable au moi idéal du militant (en tant que militaire), mais aussi la possibilité de s'en remettre à autrui dans l'expérience même de la subjectivité (une dimension de la prison toujours occultée par la société en voie de transformation). Or, c'est justement cette part qui est mise en valeur dans les expériences de prison racontées par des femmes : une zone à laquelle on a ôté toute possibilité dans le système symbolique focalisé sur les luttes politiques et s’avérant soustraite du langage. En effet, il s'agit d'affects primaires qui sont loin de pouvoir produire des valeurs positives (ces zones d'affect partent d'une exposition à l'autre non-politisé, mais potentiellement transgresseur). Creuser cette zone permettrait, peut-être, d'aborder d'une manière nouvelle les discusion actuelles sur les militants ousurceque l'on appelle la raison ilitate a partir d'une interogation militante à partir dune interrogation sur lexpérience de ceux qui se retrouvent en dehors des récits les plus diffusés. En particulier concernant un type singulie de récits de prison, que je propose de considérer ici comme « constellation » : il s'agit de femmes qui tentent de créer un espace pour que surgissent des images de liberté, cette dernière se projetant alors comme potentialité (idéaux de liberté) et non comme simple témoignage sur le passé. Leurs récits se situent dans une sorte d'entre-deux de l'écriture où le politique est posé autrement: on le suspend, on l'ouvre et on le questionne à partir d'un langage du collectif. Il est intéressant de noter, à ce titre, que pendant et après la coexistence en prison avec d'autres prisonnières, l'expérience politique se modifie profondémentet provoque un tournant dans l'idée de subjectivité, d'affect et de politique qui n'existait pas avant la prison et contient une conscience de genre. Ainsi, Fabbri écrit.

[...] lorsque je cherche un fil de continuité, je vois la jeune femme soutenue, pendant des années, par ce réseau invisible de solidarités complices. Je sais que je ne suis pas en train de parler de politique, parce que cela ne me plât pas, et je sais aussi que je ne suis pas en train de parler de sentiments. Je suis en train de parler du fait que nous vivions ensemble, des aspects quotidiens et de l'extrême. Je me souviens toujours de la salle d'eau.

Quelques pages plus loin, elle précise que ce passé ne répond pas au registre héroïque :

Ça n’a pas été une vie héroïque, du moins pas dans le sens où elle était construite autour d'actes d'héroïsme. Il est clair qu'il y en a eu. Mais ce ne sont pas ceux-là que nous avons besoin de rappeler, je répète que ce n'est pas cela, ou que ce ne sont pas seulement ces choses-là qui ont maintenu notre regard intact. J'aime bien utiliser ce mot parce que je crois vraiment que quelque chose en nous demeure intact ${ }^{15}$.

Cet extrait offre des clés pour comprendre le problème que postule l'écrivain par rapport au témoignage comme épopée (politique) ou mémoire subjectiviste. Il est curieux qu'un texte qui parle de la jeune militante emprisonnée se situe en dehors de la politique et des sentiments, deux topoï qui convoquent les formats les plus de la politic plus communs du témoignage des victimes. Par ailleurs, le souvenir et l'oubli apparaissent comme une construction à laquelle s'adosse le récit du passé - écoute du passé à partir du présent. De ce point de vue, la critique de la victimisation postule un autre rapport au passé, à savoir une attitude d'écoute, à la fois geste, acte et commentaire:

Il serait facile de dire que jécris contre l'oubli, mais je n'y crois pas. Il y a aussi un droit à l'oubli. Un droit à se méfier des souvenirs. Je ne sais pas si on écrit pour oublier ou pour rappeler. Mais c'est contre quelque chose, contre ce que d'autres écrivent ou ce que d'autres passent sous silence. Peut-être aussi, contre son propre silence, contre les souécrit il y a peu de temps que «Oblivion ressemble au pardon ${ }^{16}$."

C'est le fait d'aborder le souvenir par-delà les connotations morales qui détermine, dans Oblivion un rapport créatif au passé mettant en jeu un affect qui n’appartient pas à la catégorie du politique ni à celle du sentimental. Lorsque Fabbri signale que ce passé n’est pas du tout « héroïque » en dépit de certains actes d'héroïsme, elle nous invite à différencier entre l'acte et la capacité, l'acte et la puissance. Lacte de mémoire est un droit, mais oblivion (du latin : obliviscor, oublier) est une capacité, une potentialité ouvrant la perspective d'un dialogue différent entre le passé et le présent. Cela implique un changement d'attitude à l'égard du sujet qui manifeste cette capacité et une prise de distance vis-à-vis de l'image de la « victime » dont on met en avant le statut pasif la douleur t' l'impuissonce. Il ne s' meten avent lo tomber dans une moralsationde la gestes qu aident à penser autres stigmates dans le souvenir de la dictature. Une écriture postulée comme processus qui, s'interrogeant sur elle-même et ses propres implications, devient une façon d'intervenir sur le souvenir en ouvrant une sorte de théâtre qui nous éloigne des automatismes de la perception concernant les victimes. Une telle écriture nous invite à penser la possibilité de nouvelles manières de concevoir le passé adressées aux personnes qui n’ont pas vécu l'expérience de l'emprisonnement, et l'émergence de nouveaux sens que la reconstruction de ce passé peut prendre en charge dans le présent.
(15) Edda Fabbri, Oblivion, op (16) 